\title{
25 years of audiovisual cartography and the research continues
}

\author{
Dennis Edler ${ }^{1}$
}

Published online: 1 March 2019

(c) The Author(s) 2019

The opportunities to visualize spatial phenomena with multimedia elements have become highly versatile. Since the publication of John B. Krygier's typology of abstract sound variables in 1994, cartographers have begun to explore the multitude of methods and techniques to create and publish examples of audiovisual maps for a diverse range of thematic applications. These maps deal with questions of many spatial disciplines, such as geography, geology, and environmental sciences. They include abstract sounds, sound sequences recorded in the real environment (audiorealistic soundscapes), recorded language or music to enrich the visual dimension. Sound is used redundantly to strengthen visually communicated information, it can also represent additional information which is not a part of the map graphics.

In the past few years, cartography has received new impetus through neighboring disciplines and related industries, such as informatics and video and computer gaming. The continuous further development of core technologies of the world wide web, such as programming languages, as well as the growing field of creating individual virtual environments in virtual and augmented reality (VR/AR) have made it possible to construct 2D and 3D maps with new approaches of animation and interaction. Today, cartographers are free to use software products which have not been in the focus of more traditional multimedia map-making.

It becomes obvious-again-that new technologies attract attention and provoke people interested in cartography and visualization to test out methods and techniques of creating vivid and vibrant spatial media. It seems 'natural' that the auditory dimension is given a high importance when trying to explore new options to create highly realistic cartographic visualizations. This special issue of the newly shaped KN journal is dedicated to modern approaches of audiovisual map design. The contributions indicate the potential of modern visualization techniques for the future of multimedia cartography. The articles address several main topics of audiovisual cartography: the development of audiovisual cartography so far, modern methods of embedding sound in VR landscapes and in web and mobile mapping applications as well as the importance of user-centered cartography considering the cognitive processing of cartographically represented spatial information.

Enjoy reading!

Dennis Edler

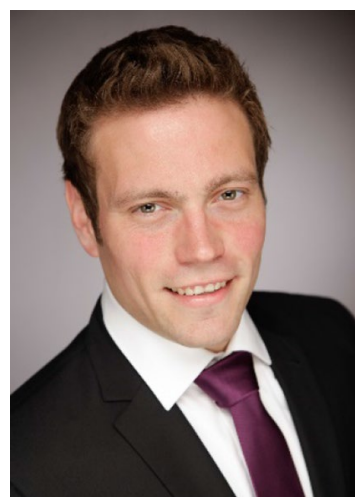

Open Access This article is distributed under the terms of the Creative Commons Attribution 4.0 International License (http://creativeco mmons.org/licenses/by/4.0/), which permits unrestricted use, distribution, and reproduction in any medium, provided you give appropriate credit to the original author(s) and the source, provide a link to the Creative Commons license, and indicate if changes were made.
Dennis Edler

dennis.edler@rub.de

1 Geomatics/Cartography Group, Geography Department, Ruhr University Bochum, Bochum, Germany 